\title{
SUBGRID-SCALE CLOSURE FOR THE INVISCID BURGERS-HOPF EQUATION*
}

\author{
STAMEN I. DOLAPTCHIEV ${ }^{\dagger}$, ILYA TIMOFEYEV ${ }^{\ddagger}$, AND ULRICH ACHATZ ${ }^{\S}$
}

\begin{abstract}
A method is presented for constructing effective stochastic models for the timeevolution of spatial averages in finite-difference discretizations of partial differential equations. This method relies on the existence of a time-scale separation in the dynamics of the spatial averages and fine-grid variables. The spatial averages, thus, are treated as the slow variables in the system and a stochastic mode reduction strategy can be applied to derive a closed form effective stochastic model. A conservative discretization of the Burgers-Hopf equation is used as a particular example to illustrate the approach. An advantage over previous applications of stochastic mode reduction to spectrally discretized models is that the resulting closure is local and thus remains applicable even if the number of slow variables is large.
\end{abstract}

Key words. Stochastic parameterization, coarse-grained modeling, stochastic mode elimination.

AMS subject classifications. 60J60, 60H10, 62M99.

\section{Introduction}

Reduced stochastic modeling in spatially extended systems has been an important research topic for many decades, with atmospheric sciences being one of the major application areas. In particular, reduced models describing the behavior of leading patterns of atmospheric variability $[24,3,4,1,2,14]$ present a viable alternative in reducing the computational complexity of coupled atmosphere-ocean models. In the last few decades reduced modeling in spatially extended systems received an increased attention in the mathematical community as well. In particular, various techniques have been proposed to model the behavior of essential degrees of freedom in different applied settings. For instance, such techniques include derivation of reduced dynamics in multiscale stochastic differential equations (SDEs) [16, 8], optimal prediction [5, 6], reduced Markov chain modeling [19, 7, 12, 22], and empirical estimation of linear reduced models $[4,13]$.

In this paper we apply a stochastic mode reduction strategy, proposed by Majda, Timofeyev, and Vanden-Eijnden $[16,17,21]$ and known as MTV, to derive an effective stochastic model for spatial averages in a finite-difference or finite-volume discretization of partial differential equations. Such discretizations are widely used in ocean modeling, regional atmosphere modeling and more recently in global atmosphere models. The coupled atmosphere-ocean system presents considerable computational challenges since the two components evolve on vastly different time-scales. The atmospheric component is much faster and requires a much smaller time-step, and thus typically consumes more of the computational time. The approach presented in this paper is designed to eliminate this limitation by considering effective stochastic models for atmospheric discretizations averaged over some pre-defined spatial box. The subgrid-scale closure is local, i.e. its effect on some local average only

*Received: February 21, 2012; accepted (in revised form): August 23, 2012. Communicated by David Cai.

${ }^{\dagger}$ Institut für Atmosphäre und Umwelt, Goethe-Universität Frankfurt, Altenhöferallee 1, 60438 Frankfurt am Main, Germany (dolaptchiev@iau.uni-frankfurt.de).

${ }^{\ddagger}$ Department of Mathematics, University of Houston, Houston, Texas, USA (ilya@math.uh.edu).

§Institut für Atmosphäre und Umwelt, Goethe-Universität Frankfurt, Altenhöferallee 1, 60438 Frankfurt am Main, Germany (achatz@iau.uni-frankfurt.de). 
needs information from the immediate neighborhood. Note that in former applications of stochastic mode reduction to spectrally discretized models [11, 15, 10, 18], the information from all resolved modes is always required. In contrast to these, a SGS closure as proposed here does not become impracticable if the number of resolved modes is large. The new method bears thus the potential for application in turbulence parameterization in the context of large eddy simulations.

To construct the effective model we introduce a coarse grid and define new dynamic variables as averages of the sub-grid scales over the coarse box. The sub-grid scales are equivalent to the fine-grid variables in this context. Next, we apply the stochastic mode reduction to eliminate the subgrid-scale variables from the equation. To mimic the properties of some conservative finite-difference/finite-volume discretizations, we apply our methodology to a prototype chaotic system [20], obtained as a finite-difference discretization of the inviscid Burgers-Hopf equation. We demonstrate that the stochastic mode reduction technique can be successfully utilized to obtain an effective stochastic model for spatial averages over the coarse grid. In particular, we compare and contrast the statistical behavior of the new coarse-grained model with the one from the full model (direct numerical simulation) and from two purely empirical coarse-grained models.

The outline of this paper is as follows: in Section 2 we introduce the discrete version of the inviscid Burgers model. In Section 3 we discuss the stochastic mode reduction technique and present a new reduced model with stochastic subgrid-scale closure. The performance of this model is assessed in Section 4 by comparing it with the full model and with two other coarse-grained models with purely empirical Ornstein-Uhlenbeck type closures. A summary and a conclusion are presented in the last section.

\section{The inviscid Burgers-Hopf equation}

2.1. The discrete model. We consider here the one dimensional inviscid Burgers-Hopf equation

$$
\frac{\partial u}{\partial t}+\frac{\partial}{\partial x} \frac{u^{2}}{2}=0
$$

over a periodic domain of size $L$. Discretizing equation (2.1) in space, one can write

$$
\frac{d}{d t} u_{i}+\frac{F_{i+\frac{1}{2}}-F_{i-\frac{1}{2}}}{\Delta x}=0
$$

where $u_{i}$ denotes the values of the function $u$ at the center of the discrete cell $i(i \in s=$ $\{0, \ldots, N-1\}$ ) of width $\Delta x=\frac{L}{N}$ and $F_{i+\frac{1}{2}}, F_{i-\frac{1}{2}}$ denote the fluxes at the boundaries of cell $i$. These fluxes must be approximated using values from neighboring cells; we use the discretization proposed in [27]:

$$
\begin{aligned}
& F_{i+\frac{1}{2}}=\frac{1}{6}\left(u_{i+1}^{2}+u_{i} u_{i+1}+u_{i}^{2}\right), \\
& F_{i-\frac{1}{2}}=\frac{1}{6}\left(u_{i}^{2}+u_{i} u_{i-1}+u_{i-1}^{2}\right) .
\end{aligned}
$$

The above discretization conserves total momentum, total energy, and the Liouville property. We refer to [20] for an analysis of the mathematical properties of the finite-difference scheme. The numerical approximations presented in [20] are characterized by strong chaos with ergodic behavior. This motivated us to use the 
discretized equation (2.2) with (2.3), (2.4) as a test model for the stochastic mode reduction procedure.

A typical problem, encountered in modeling processes involving interactions on a wide range of spatial scales, is that one cannot afford to solve the discrete model on a sufficiently small grid, but can afford to solve it on some much coarser grid. The model equations must be reformulated in terms of resolved and unresolved (subgrid-scale) variables. In large eddy simulation this is done by averaging the equations. Following the volume-balance procedure of [23] we average over $n$ neighboring, fixed in space, "fine" grid cells of the original width $\Delta x$. We define a "coarse" grid with a spacing of $n \Delta x$ and cell index set $s_{x}=\left\{0, \ldots, N_{x}-1\right\}$, where $N_{x}=\frac{N}{n}$ is the total number of coarse cells $(N$ a multiple of $n)$. The configuration of the coarse and fine grid cells is depicted in figure 2.1. Let $x_{i}, i \in s_{x}$ denote the averaged value of $u_{i}, i \in s$ on the coarse grid; $x_{i}$ is computed by applying a top-hat filter to $u_{i}$. This filtering can be written simply as the arithmetic mean over $n$ neighboring grid cells

$$
x_{i}=\frac{1}{n} \sum_{k=n i}^{n(i+1)-1} u_{k} .
$$

Next, we split $u_{i}$ into a mean $x_{\hat{i}}$ and a deviation $y_{i}$

$$
u_{i}=x_{\hat{i}}+y_{i},
$$

where the index $\hat{i}$ denotes the coarse cell in which the fine cell $i$ is located. We will refer to $x_{\hat{i}}$ and $y_{i}$ as the resolved and unresolved modes, respectively. Applying the averaging operator (2.5) to the discretized Burgers-Hopf equation, equation (2.2) can be written in terms of $x_{\hat{i}}$ and $y_{i}$ :

$$
\begin{aligned}
& \frac{d}{d t} x_{\hat{i}}+\frac{F_{n(\hat{i}+1)-\frac{1}{2}}-F_{n \hat{i}-\frac{1}{2}}}{n \Delta x}=0, \\
& \frac{d}{d t} y_{i}+\frac{F_{i+\frac{1}{2}}-F_{i-\frac{1}{2}}}{\Delta x}-\frac{F_{n(\hat{i}+1)-\frac{1}{2}}-F_{n \hat{i}-\frac{1}{2}}}{n \Delta x}=0 .
\end{aligned}
$$

The flux terms in (2.7) and (2.8) contain both resolved and unresolved modes. We split these terms depending on the modes involved and obtain the following system of ODEs for the discretized model equations:

$$
\begin{aligned}
& \dot{x}_{i}=\sum_{j \in s_{x}} \sum_{k \in s_{x}} B_{i j k}^{x x x} x_{j} x_{k}+\sum_{j \in s_{x}} \sum_{k \in s} B_{i j k}^{x x y} x_{j} y_{k}+\sum_{j \in s} \sum_{k \in s} B_{i j k}^{x y y} y_{j} y_{k}, \quad i \in s_{x}, \\
& \dot{y}_{i}=\sum_{j \in s_{x}} \sum_{k \in s_{x}} B_{i j k}^{y x x} x_{j} x_{k}+\sum_{j \in s_{x}} \sum_{k \in s} B_{i j k}^{y x y} x_{j} y_{k}+\sum_{j \in s} \sum_{k \in s} B_{i j k}^{y y y} y_{j} y_{k}, \quad i \in s .
\end{aligned}
$$

The terms of the form $B^{\alpha \beta \gamma}$ arise from the quadratic nonlinearities; the first superscript $\alpha$ indicates the modes on which they project, and the second two, $\beta$ and $\gamma$, indicate the two modes involved. In (2.9), (2.10) the indices of the summation signs go formally over $s$ or $s_{x}$; however, one must keep in mind that due to the local form of the discretization the tensors with the interaction coefficients are sparse and involve only "neighboring" indices. The explicit form of the tensors can be found in Appendix A. 


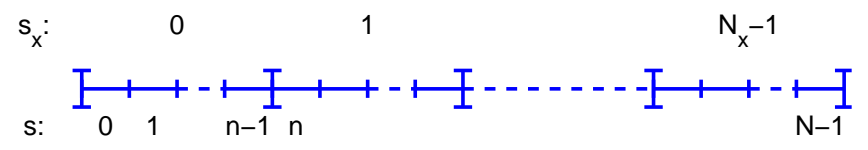

FIG. 2.1. Schematic representation of the fine grid cells (small intervals) and coarse grid cells (large intervals) introduced in Section 2.1. The indexing of the fine/coarse cells is denoted by the integers corresponding to $s / s_{x}$, respectively.

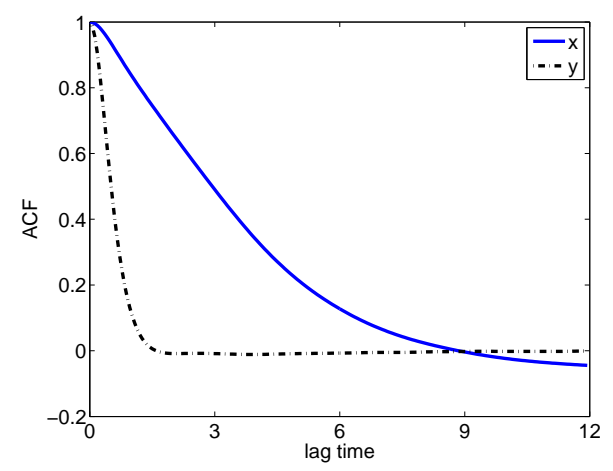

FIG. 2.2. Time autocorrelation functions for the resolved $(x)$ and unresolved $(y)$ modes from the DNS. The averaging interval $n$ is set to 16; the time unit on the abscissa is 20 nondimensional model time units.

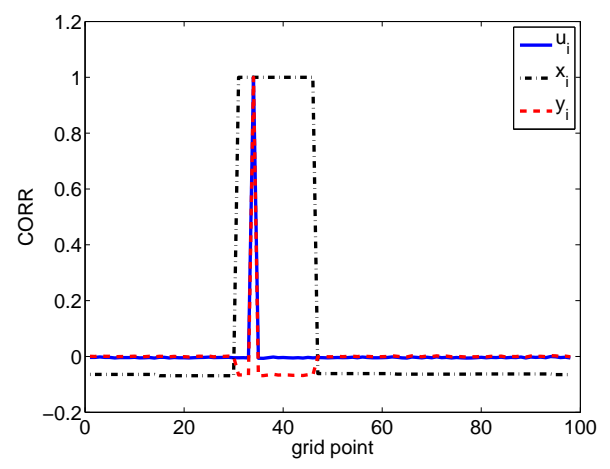

FIG. 2.3. Spatial correlation from the DNS of $u_{i}$, resolved mode $x_{i}$, and unresolved mode $y_{i}$, all for an arbitrary chosen grid point $i=36$. The averaging interval $n$ is set to 16; grid points with $i=32, \ldots, 48$ are located inside the same coarse cell.

2.2. Statistics in the full model. Equations (2.9), (2.10) represent another form of the discretized model (2.2), (2.3), (2.4); we will refer to this model as the full model and to its integration as the direct numerical simulation (DNS). We integrate the full model in time using a 3rd order Runge-Kutta method with the following choice of parameters: $\Delta t=0.02, L=100, N=256$. The initial condition represents a random distribution of $u$, characterized by zero total momentum and a total energy of 1.716 .

The time autocorrelation functions of the $x$ - and $y$-modes are presented in fig- 
ure 2.2; the averaging interval $n$ from (2.5) is set to 16 . Because of the spatial homogeneity of the model, all statistical moments in the paper represent a spatial average. From the figure it is visible that the $x$-modes evolve on a slower time scale than the $y$-modes. By computing the area under the curves, we estimated decay time scales of 66 and 12 for the $x$ - and $y$-modes, respectively. This implies a moderate scale separation in time of about 5.5. The scale separation is proportional to the averaging interval $n$ : for larger values of $n$ the $x / y$-modes become slower/faster.

In figure 3.1(b) the autocorrelation function of the $x$-modes from the DNS for larger lags is displayed. We observe oscillations in the tail with periods much larger than the estimated decay time scale. It was found that the magnitude of these oscillations depends on $n$ as well. Long-period oscillations are visible in the tails of all of the DNS autocorrelation functions reported in the paper. Although characterized by small amplitudes, these oscillations appear to be an inherent property of the low-frequency dynamics of the resolved modes.

Figure 2.3 depicts the spatial correlation pattern in the DNS. We see that the full variable $u_{i}$ is uncorrelated in space. The subgrid-scale variable $y_{i}$ is also, to a good approximation, uncorrelated, except of small negative correlation values for grid points corresponding to the same coarse cells. This is due to the constraint that the $y_{i}$ 's sum to zero inside the coarse cell. From the pattern of $x_{i}$ we can conclude that the correlations between different coarse cells are negligible.

\section{Stochastic mode reduction}

The stochastic mode reduction $[16,17,21,18]$ can be thought of as a three step procedure. In the first step, one has to impose certain assumptions on the statistical behavior of the full model (2.9), (2.10). In particular, there should exist a time-scale separation (at least moderate) between the resolved and unresolved variables. Essentially this means that we can treat the resolved and unresolved variables as the slow and fast degrees of freedom, respectively. For the discrete model we consider, this assumption is verified in Section 2.2 and figure 2.2. In addition, the stochastic mode reduction also requires that the term $B_{i j k}^{y y y} y_{j} y_{k}$ is the most dominant term in the equation for fast variables. The second step in the stochastic mode reduction procedure is a stochastic modeling step for the term $B_{i j k}^{y y y} y_{j} y_{k}$ (see (3.1)). This step allows explicit analytical calculations of the coefficients in the reduced model. Finally, the third step consists of further accelerating the fast variables, which allows one to compute the reduced equations in the limit of infinite time-scale separation. Typically, the full model under consideration has only a moderate separation of time scales between the fast and slow variables. Nevertheless, the mode reduction procedure has been successfully tested on many models with moderate time-scale separation, which suggests that often the slow variables are not particularly sensitive to the time-scale of the fast modes. The third step can be formalized by introducing an explicit scale separation parameter $\varepsilon$ into the equations. This yields a modified (also called selectively accelerated [18]) model of the same dimensionality as the original full model. One then can test the existence of the limit of the modified model as $\varepsilon \rightarrow 0$.

If the self-interactions of the slow variables in the equation for slow variables (the term $B_{i j k}^{x x x} x_{j} x_{k}$ ) are absent in the full model, then the modified model can also be obtained by introducing a coarse-grained time $t \rightarrow \varepsilon t$. On the other hand, if these terms are non-zero then the time rescaling alone will not yield the modified equations in the proper form, and an additional assumption on the behavior of these terms must be imposed. In particular, one must assume that the self-interactions are rescaled as $B_{i j k}^{x x x} x_{j} x_{k} \rightarrow \varepsilon B_{i j k}^{x x x} x_{j} x_{k}$ under the time rescaling $t \rightarrow \varepsilon t$ (assumption A3 on p.896 in 
[16]). This assumption ensures that the self-interactions of the slow variables are not affected by the stochastic mode reduction. One can motivate this by the fact that the resulting reduced model is compared directly with the original full model. Moreover, the behavior of the modified model for different values of $\varepsilon$ is also compared with the behavior of the full model. Thus, a problem can potentially arise because the full model and the modified model are compared on different time-scales, but the self-interactions of the slow variables should not be affected by this time-scale difference.

Another way of looking at the introduction of different powers of $\varepsilon$ in front of different terms in the modified model is as a modeling step in order to carry out the stochastic mode reduction strategy. Different powers of $\varepsilon$ in front of various terms are necessary to take into account the different time scales and magnitudes of these terms. For instance, since we assume that the fast variables are primarily driven by the self-interactions of fast modes, these should be the leading terms in the equation for the $y$-variables. Next, since the stationary averages $\left\langle B_{i j k}^{x x y} x_{j} y_{k}\right\rangle$ and $\left\langle B_{i j k}^{x y y} y_{j} y_{k}\right\rangle$ are zero, $\varepsilon^{-1}$ is required in front of these terms in order to obtain nontrivial stochastic corrections from the stochastic mode-reduction procedure. Thus, $\varepsilon^{-1}$ is also required in front of the $B_{i j k}^{y x x} x_{j} x_{k}$ and $B_{i j k}^{y y x} y_{j} x_{k}$ terms to preserve the conservation of energy. Finally, since the full model is directly compared with the stochastically parametrized model and the reduced model (derived in the limit $\varepsilon \rightarrow 0$ ), the nonlinear self-interactions of the slow variables $B_{i j k}^{x x x} x_{j} x_{k}$ should not be affected by the stochastic mode-reduction.

Therefore, introducing $\varepsilon$ can be considered as a technical step to emphasize the influence of various terms in the equations when the fast variables are accelerated even further. On the other hand, this step should also be consistent with the structure of the equations (e.g. the conservation of energy). Finally, the original full-model is compared directly with the modified model (the stochastically parametrized model in $(3.2),(3.3))$ and the reduced model, and this is often referred to as "setting $\varepsilon$ to one".

3.1. Closure assumptions. The stochastic mode reduction procedure assumes that the term $B_{i j k}^{y y y} y_{j} y_{k}$ in (2.10) can be modeled as an Ornstein-Uhlenbeck (OU) process in $y$ :

$$
\sum_{j \in s} \sum_{k \in s} B_{i j k}^{y y y} y_{j} y_{k}=-\gamma y_{i}+\sigma \dot{W}_{i}
$$

Here $\gamma$ and $\sigma$ denote the drift and diffusion coefficient of the OU process and $W_{i}$ is a Wiener process. Because of the spatial homogeneity of the model, $\gamma$ and $\sigma$ do not show any spatial variations (are not dependent on the index $i$ ).

By introducing (3.1) we approximate the fast self-interactions between subgridscale modes only in the equation for the $y$-modes. This is in contrast with traditional subgrid-scale parameterizations, where the terms involving subgrid-scale modes in the evolution equation for $x$ are expressed in terms of resolved modes by assuming some particular closure. In our approach, the explicit form of this closure is derived by the MTV stochastic mode reduction procedure and is rigorously valid in the limit of infinitely large time scale separation between the resolved and subgrid-scale modes. We want to stress that the OU process from (3.1) does not couple neighboring subgridscale modes, since on longer time scales these modes are uncorrelated; see figure 2.3. It is thus in line with the MTV stochastic mode reduction strategy which presupposes a diagonal OU process. As shown in [9], interactions between different subgrid-scale 
modes can be incorporated in the ansatz (3.1) by choosing, instead of a scalar drift coefficient, a drift matrix.

Assuming (3.1) and properly introducing $\varepsilon$ in (2.9), (2.10), we obtain the following modified model:

$$
\begin{aligned}
d x_{i} & =\left(B_{i j k}^{x x x} x_{j} x_{k}+\frac{1}{\varepsilon} B_{i j k}^{x x y} x_{j} y_{k}+\frac{1}{\varepsilon} B_{i j k}^{x y y} y_{j} y_{k}\right) d t, \\
d y_{i} & =\left(\frac{1}{\varepsilon} B_{i j k}^{y x x} x_{j} x_{k}+\frac{1}{\varepsilon} B_{i j k}^{y x y} x_{j} y_{k}-\frac{1}{\varepsilon^{2}} \gamma y_{i}\right) d t+\frac{1}{\varepsilon} \sigma d W_{i} .
\end{aligned}
$$

In the equations above and in the following paragraphs we make use of Einstein's summation convention, and $\varepsilon \ll 1$ is a small parameter which is a measure of the scale separation in time between the $x$ and $y$ modes. We will refer to the simulation with the modified model from (3.2), (3.3) as the OU-DNS.

Finally, we want to comment on the estimation of the OU parameters $\gamma$ and $\sigma$. These parameters are determined using a time series of $y_{i}$ from the DNS and assuming that the $\mathrm{OU}$ closure dominates in (3.3). In this case $\gamma$ can be estimated (see e.g. $[17,21]$ ) as the reciprocal of the area under the graph of the modulus of the autocorrelation function for $y_{i}$. Then, the diffusion coefficient $\sigma$ is found from the variance of $y_{i}$ (denoted with $\left\langle y_{i} y_{i}\right\rangle$ ) by using that in equilibrium,

$$
\left\langle y_{i} y_{i}\right\rangle=\frac{\sigma^{2}}{2 \gamma} .
$$

The quality of this estimate is discussed in the next section.

3.2. Numerical tests of the closure assumption. We have assessed the approximation made in (3.1) by running the OU-DNS with $\varepsilon=1$ and comparing the results with the DNS. The autocorrelation functions for the $x$ - and $y$-modes from the two simulations are depicted in figure 3.1. The structure of the autocorrelation function of the subgrid-scale modes from the DNS is approximated in the OU-DNS by an exponential with similar decay rate; see figure 3.1 (a). The OU-DNS is not able to reproduce the long-period oscillations in the autocorrelation function of $x$ from the DNS; see figure 3.1 (b). However, it can roughly capture the large-scale envelope of it. Table 3.1 shows that the OU closure is able to reproduce the variance of the subgrid-scale modes and captures reasonably well the variance of the resolved modes.

The reduced stochastic model introduced in Section 3.3 represents the asymptotic limit $\epsilon \rightarrow 0$ of the OU-DNS (3.2), (3.3). This limit corresponds to infinitely large time scale separation between the $x$ - and $y$-modes. In order to study the asymptotic limit, we performed numerical simulations with (3.2), (3.3) by systematically decreasing the value of $\varepsilon$ and thus accelerating the $y$-modes. The corresponding autocorrelation functions of $x$ are displayed in figure 3.2. It is evident that as $\varepsilon$ decreases, the autocorrelation function remains close to the $\varepsilon=1$ run (note the narrow time window chosen in figure 3.2, in order to make differences visible). Further, the deviations between $\varepsilon=0.1$ and $\varepsilon=0.5$ are smaller than those between $\varepsilon=0.5$ and $\varepsilon=1$. Similar convergence is also observed in the variance of $x$. The numerical results suggest that, after the mode elimination procedure, the resulting reduced model for $x$ will behave similarly to the OU-DNS with $\varepsilon=0.1$, which is close to the modified model with $\varepsilon=1$.

3.3. The reduced stochastic model with subgrid scale closure. The stochastic mode reduction procedure allows us to eliminate the subgrid-scale modes 


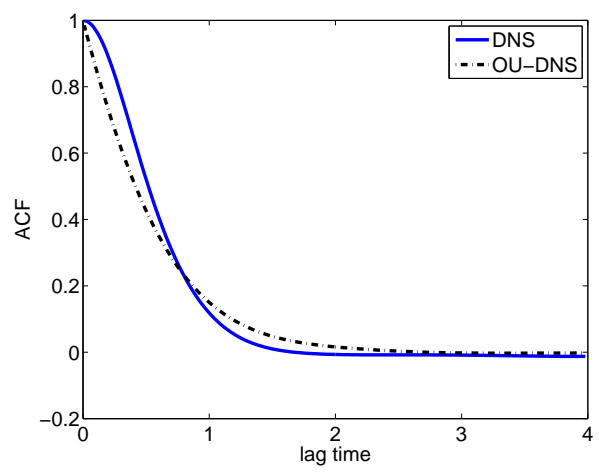

$\mathrm{b}$

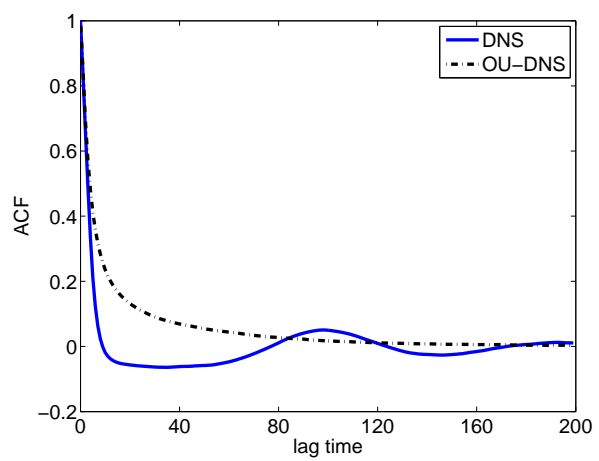

FIG. 3.1. Time autocorrelation function of unresolved modes (a) and resolved modes (b) from the DNS and the $O U-D N S$ with $\varepsilon=1$. Averaging interval and time unit on the abscissa as for figure 2.2.

\begin{tabular}{ccc}
\hline Variance & $y$ & $x$ \\
\hline \hline DNS & $1.2616 \times 10^{-2}$ & $7.8692 \times 10^{-4}$ \\
OU-DNS $\varepsilon=1$. & $1.2619 \times 10^{-2}$ & $7.2649 \times 10^{-4}$ \\
rel. error & $<0.01$ & 0.08 \\
\hline
\end{tabular}

TABLE 3.1. Variance from the DNS and the OU-DNS.

in the OU-DNS (3.2), (3.3) and derive effective equations for the resolved modes only. The procedure is carried out by performing an asymptotic expansion in the Kolmogorov backward equation corresponding to the SDEs (3.2), (3.3); see [16]. The resulting coarse-grained model reads

$$
\begin{gathered}
d x_{i}(t)=\sum_{j, k=i-1}^{i+1} B_{i j k}^{x x x} x_{j} x_{k} d t+\sum_{j=i-1}^{i+1} M_{i j} x_{j} d t+\sum_{j, k, l=i-2}^{i+2} C_{i j k l} x_{j} x_{k} x_{l} d t \\
+\sum_{j=i-\frac{1}{2}}^{i+\frac{1}{2}} P_{i j} d W_{j}^{(1)}+\sum_{j=i-\frac{1}{2}}^{i+\frac{1}{2}} D_{i j}(x) d W_{j}^{(2)}
\end{gathered}
$$

where $W_{j}^{(1)}, W_{j}^{(2)}$ denote independent Wiener processes. We will refer to (3.5) as the reduced stochastic model. The first term on the right hand side of (3.5) describes the bare truncation model, which is derived from the full model when all subgridscale modes are neglected. The other terms in (3.5) arise from the subgrid-scale model; they represent linear and cubic deterministic corrections as well as additive and multiplicative noise (last two terms). Note the local form of the subgrid-scale model as visible from the summation intervals. This is in contrast to the heretofore applications of MTV to spectrally discretized models. There the closure for any resolved mode involves all other resolved modes. It thus becomes impracticable as the number of resolved modes gets too large. The explicit form of the subgrid-scale parameterization is given in Appendix B. The reduced stochastic model conserves 


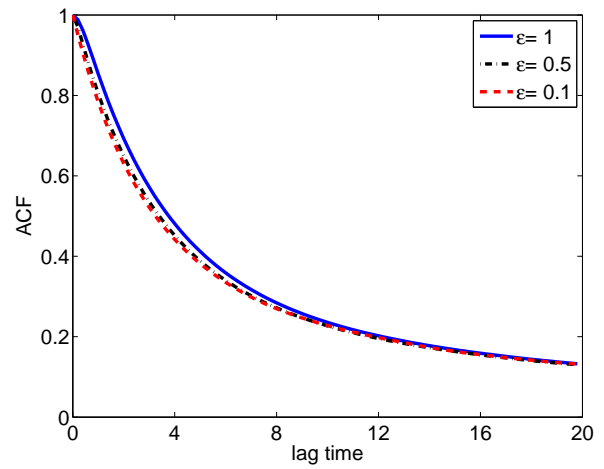

FIG. 3.2. Time autocorrelation function of the resolved modes from the OU-DNS for different values of $\varepsilon$. Averaging interval and time unit on the abscissa as for figure 2.2.

\begin{tabular}{cccccc}
\hline Lag $k$ & 0 & 1 & 2 & 3 & 4 \\
\hline Int. CCF & 1.3149 & 0.2000 & 0.0260 & -0.0750 & -0.1325 \\
\hline \hline Lag $k$ & 5 & 6 & 7 & 8 & \\
\hline Int. CCF & -0.1721 & -0.1883 & -0.2231 & -0.2221 & \\
\hline
\end{tabular}

TABLE 4.1. Integrated normalized cross-correlation function $C_{i, i+k}(\tau) / C_{i, i}(0)$ (Int. CCF) from the DNS for different lags $k$; here $C_{i, i+k}(\tau)=\left\langle x_{i}(t) x_{i+k}(t+\tau)\right\rangle$. For $N_{x}=16$ only cross-correlations up to lag 8 are required due to the spatial homogeneity.

total momentum, as does the full model. The energy $\left(\sum_{i \in s_{x}} x_{i}^{2}\right)$ is not conserved exactly, but fluctuates around a constant mean value. This, however, is also the case in the full model, where energy is transfered between the resolved and unresolved scales.

4. Results for the reduced stochastic model

In the following section we study the performance of the reduced stochastic model by comparing it with the full model, the linear-closure stochastic model, and the stochastic model of multivariate OU type.

4.1. The multivariate $\mathrm{OU}$ model and the linear-closure stochastic model. Since the derived reduced stochastic model represents a local parameterization, it is natural to ask the question if a global closure, coupling all resolved modes, can outperform it. As a zero order global closure we consider a multivariate OU process (MVOU)

$$
d x_{i}=\Gamma_{i j} x_{j} d t+\Sigma_{i j} d W_{j},
$$

where $\Gamma, \Sigma \in \mathbf{R}^{N_{x} \times N_{x}}$ denote the drift and diffusion matrices. Empirical models of global form, similar to (4.1), are often used in atmospheric modeling; see, e.g., [26, 4]. It can be shown that the lagged cross-correlation of the process in (4.1) satisfies

$$
C(\tau)=e^{\Gamma \tau} C(0)
$$


where $C(\tau)=\left\langle\vec{x}(t) \vec{x}(t+\tau)^{T}\right\rangle$ denotes the cross-correlation matrix at lag $\tau$. By integrating (4.2) in time, the following useful relation for $\Gamma$ can be obtained:

$$
\Gamma^{-1}=-\int_{0}^{\infty} C(\tau) C^{-1}(0) d \tau .
$$

In order to find the model parameters in (4.1), first the matrices $C(0)$ and $C(\tau)$ (with different lags $\tau$ ) are estimated from a time series of the resolved variables. Then, the drift matrix $\Gamma$ is determined from (4.3), so that it matches the integrated lagged cross-correlation of the full model. Finally, the diffusion matrix $\Sigma$ is found from the fluctuation-dissipation relation to match the covariance of the full model

$$
\Gamma C(0)+C(0) \Gamma^{T}=-\Sigma \Sigma^{T} .
$$

For known $\Gamma$ and $\Sigma$ all statistical moments of the model from (4.1) can be computed analytically. The models of the type in (4.1) typically perform very well if the correlation functions of the slow variables are close to exponential. In this case, the estimation procedure described above provides damping and forcing parameters consistent with the large-scale statistical features of the slow variables, and the multivariate OU model is likely to reproduce the statistics of the slow variables. On the other hand, if the correlation functions of the slow variables are non-exponential, then the model (4.1) is more likely to fail.

While in (4.1) the non-linear self-interactions of the slow variables are not included in the effective model (as in $[26,4]$ ), there are also studies which emphasize the importance of these terms in reduced models $[1,2,25,7]$. This motivated us to consider the linear-closure stochastic model (LCSM), which is based on an ad-hoc approximation of all subgrid-scale interactions in (2.9) by an OU process with drift $\alpha$ and diffusion $\beta$ :

$$
d x_{i}=B_{i j k}^{x x x} x_{j} x_{k} d t+\alpha x_{i} d t+\beta d W_{i} .
$$

The inclusion of the non-linear self-interactions in (4.5) might improve the representation of non-Gaussian features of the full model, where the multivariate OU model from (4.1) will definitely fail. We have experimented with OU closures coupling neighboring grid cells, but it turned out that the particular choice in (4.5) (no coupling) gives nearly optimal results. The diagonal choice for the damping and diffusion in (4.5) is supported by the weak statistical coupling between different slow variables. In particular, cross-correlation times (integral of the lagged cross-correlation functions $\left.\left\langle x_{i}(t) x_{j}(t+\tau)\right\rangle\right)$ are presented in table 4.1. The behavior of the cross-correlations indicates that the coupling is weak for spatial lags greater than zero. Further, the estimate for $\Gamma$ in (4.1) reveals a diagonally dominant damping matrix. Therefore, omitting the non-diagonal terms does not have a significant affect on the model in (4.5).

By comparing the statistical behavior of the slow variables from a simulation with and without the $B_{i j k}^{x x x} x_{j} x_{k}$ in the full model (2.9), (2.10), we found that these interaction terms are rather weak and do not significantly affect the behavior of the slow variables. In particular, the marginal equilibrium distribution and the autocorrelation function are not affected by these terms (these results are not shown). This can be viewed as a numerical justification for the missing $\varepsilon^{-1}$ factor in front of the non-linear slow self-interaction terms in equation (3.2) of the modified model (see also the discussion in the beginning of Section 3). 
a

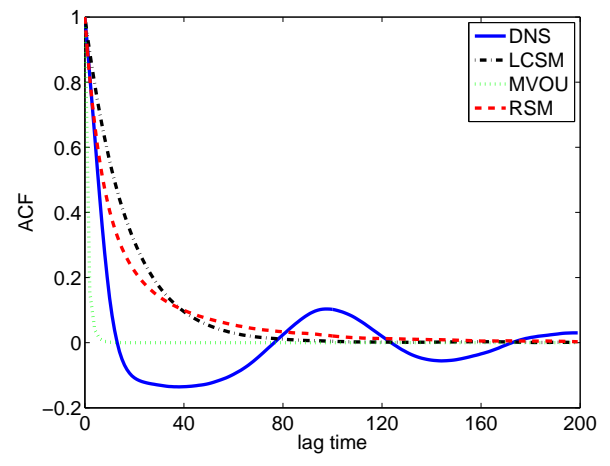

$\mathrm{b}$

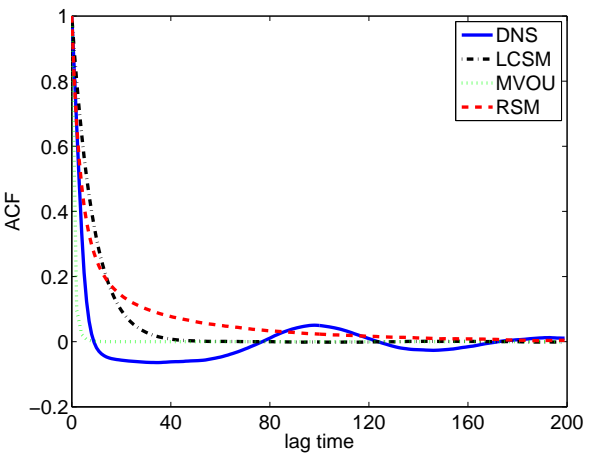

C

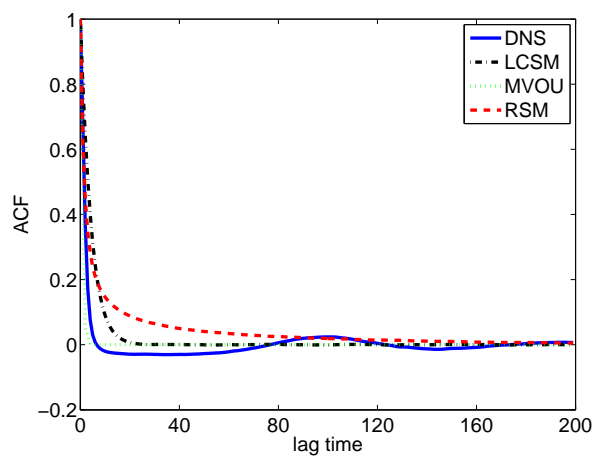

FIG. 4.1. Time autocorrelation function of the resolved modes from the DNS, linear-closure stochastic model (LCSM), multivariate OU model (MVOU), and reduced stochastic model (RSM) for $n=32$ (a), $n=16(b)$, and $n=8$ (c).

The closure parameters $\alpha$ and $\beta$ from (4.5) are estimated with the same procedure as the coefficients in (3.1) (see Section 3.1), but now from the variance and the integrated modulus of the autocorrelation function for $x_{i}$. Since the subgrid-scale terms dominate in (2.9), the estimation procedure guarantees that the linear-closure stochastic model will capture the variance and the large-scale decay behavior in the autocorrelation function from the DNS.

Overall, based on the statistical tests for the slow variables in the full model reported above, we expect the behavior of the empirical coarse-grained models (4.1) and (4.5) to be qualitatively similar, especially if the variance is considered. Differences in the simulated autocorrelation function may emerge due to the different estimation procedures for the drift coefficients.

4.2. Statistics of the reduced models. The reduced models are integrated with the parameter setup from Section 2.2. In order to study how the number of subgrid-scale modes affects the statistics of the resolved modes, we perform simulations for different averaging intervals: $n=8,16,32$. Note, that the parameters $\gamma$ and $\sigma$ from (3.1), entering in the reduced stochastic model, have been determined only once (for $n=16$ ) and then been used in all three cases. In contrast to this, the linearclosure stochastic model and the multivariate OU model require new estimation of the closure parameters for each different case. 
a

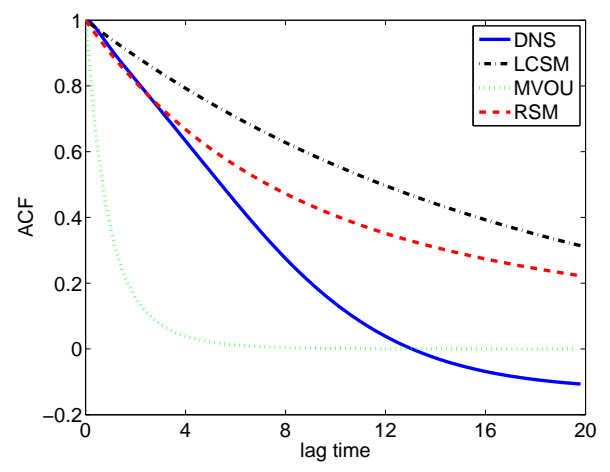

$\mathrm{b}$

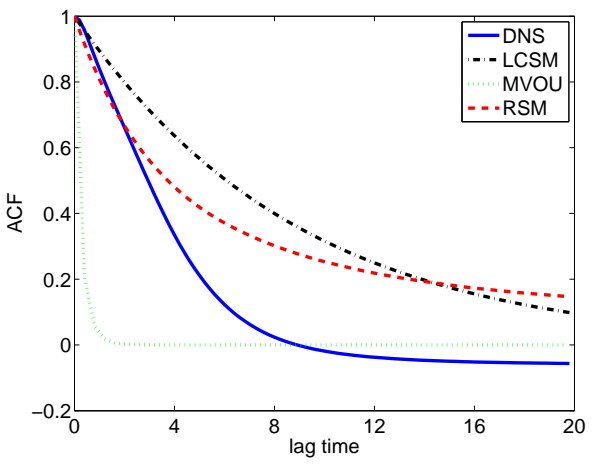

$\mathrm{c}$

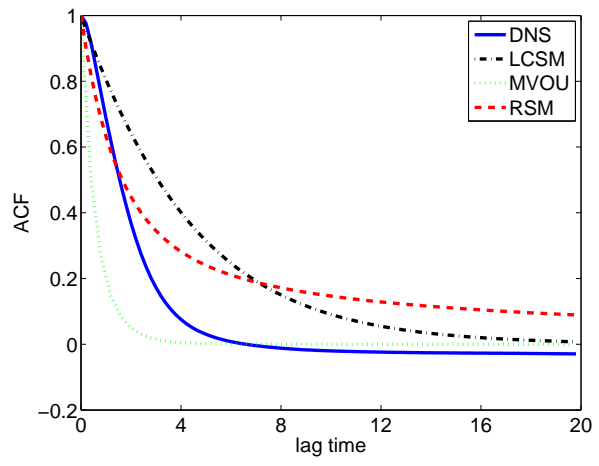

FIG. 4.2. Same as figure 4.1, but for small lags.

The autocorrelation functions of all three reduced models are shown in figure 4.1. The linear-closure stochastic model and the reduced stochastic model are able to capture only the overall decay behavior in the autocorrelation function of the DNS, the multivariate OU model completely underestimates the decay time scale. None of the models are able to reproduce the oscillations in the autocorrelation function. We take a closer look at the differences between the reduced stochastic model and the other coarse-grained models. Figure 4.2 displays the autocorrelation functions of the models for small lags. It is evident that the reduced stochastic model can capture the slope of the DNS curve at lags close to zero for all values of $n$, whereas the linearclosure stochastic model and multivariate OU model fail. In figure 4.3 a semilog plot of the absolute value of the autocorrelation functions is presented, in order to make discrepancies in the tails visible. The linear-closure stochastic model and the reduced stochastic model perform poorly for the case $n=32$. However, the reduced stochastic model captures roughly the low-frequency envelope of the DNS for $n=16$ and perfectly for $n=8$. In the latter two cases the linear-closure stochastic model is damping too strongly at large lags. Again it is evident that for all different values of $n$, the decorrelation time scale is completely underestimated by the multivariate OU model.

Figure 4.4 shows the simulated kurtosis with the models. For a given time series 
a

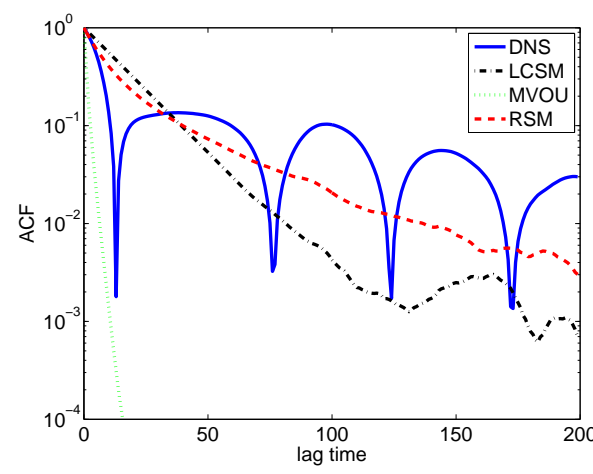

$\mathrm{b}$

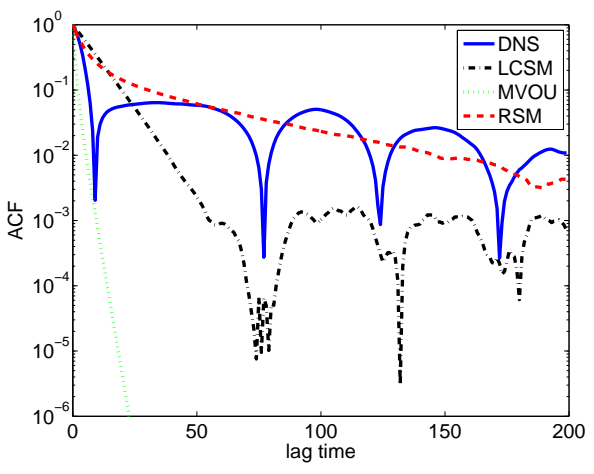

$\mathrm{C}$

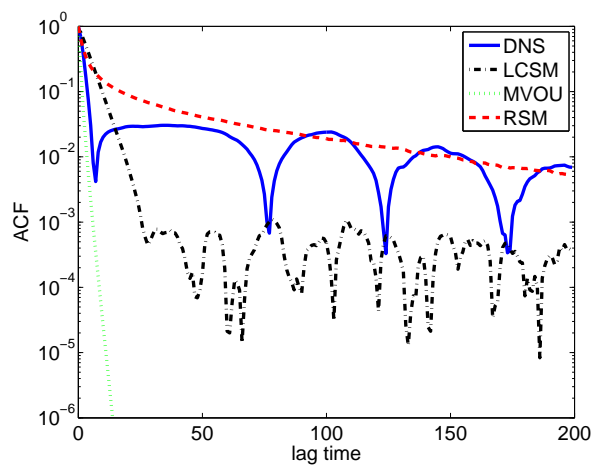

FIG. 4.3. Semilog plot of the absolute value of the time autocorrelation function of $x$ from the DNS, linear-closure stochastic model (LCSM), multivariate OU model (MVOU), and reduced stochastic model (RSM) for $n=32$ (a), $n=16$ (b), and $n=8$ (c).

$x(t)$ the kurtosis at lag $s$ is defined as

$$
K(s)=\frac{\left\langle x(t+s)^{2} x^{2}(t)\right\rangle}{\left\langle x^{2}(t)\right\rangle^{2}+2\langle x(t+s) x(t)\rangle^{2}},
$$

where $\langle\cdot\rangle$ denotes a time average. The kurtosis measures deviations from Gaussianity, since for a Gauss process it is equal to one (see the MVOU lines in figure 4.4). Looking at the DNS profiles in figure 4.4, we observe the same structure for all $n$ : a minimum of kurtosis at smaller lags, whereas at larger lags the kurtosis takes a constant value close to one. It is evident from the figures that for decreasing $n$ the magnitude of the minimum in the DNS increases, indicating weak non-Gaussianity. The reduced stochastic model is able to capture the position of this minimum and, to some extent, its magnitude. The linear-closure stochastic model completely underestimates the minimum and shifts it to much larger lags.

Deviations from Gaussian statistics in the linear-closure stochastic model simulations are entirely due to the bare truncation part in (4.5). Thus, one can conclude that the additional subgrid-scale correction terms in the reduced stochastic model, such as cubic terms and multiplicative noise, significantly improve the representation of the kurtosis. 
a

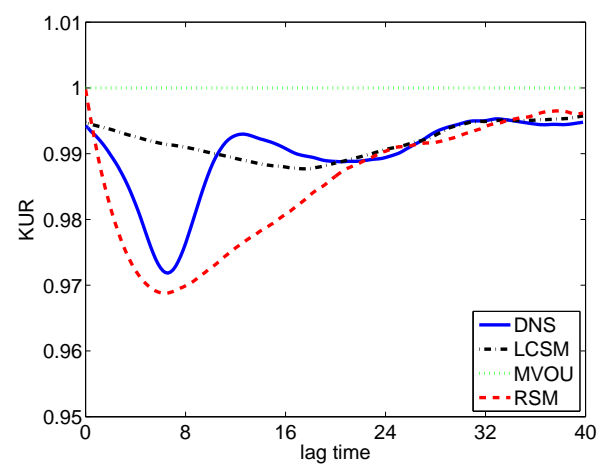

$\mathrm{b}$

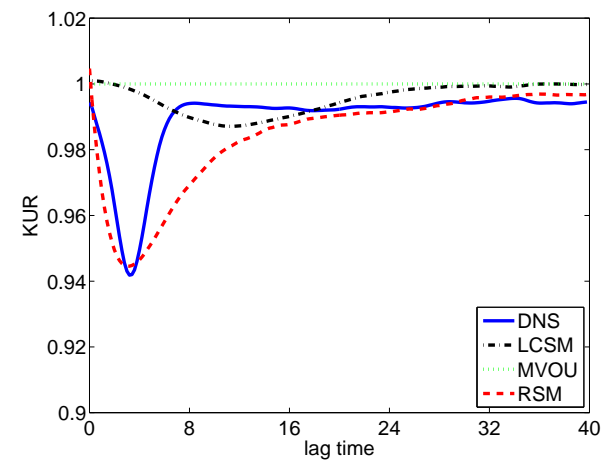

$\mathrm{c}$

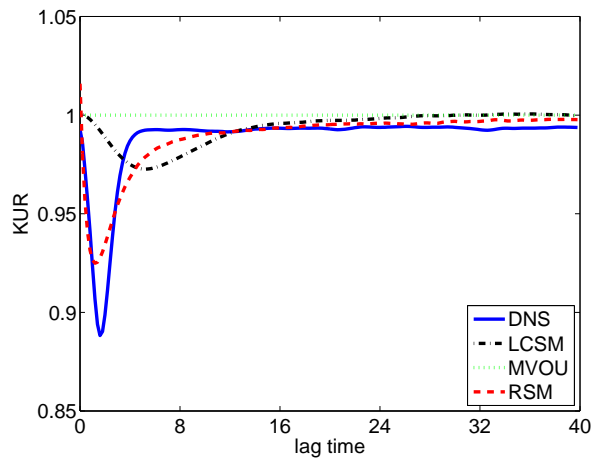

FIG. 4.4. Kurtosis of the resolved modes from the DNS, linear-closure stochastic model (LCSM), multivariate OU model (MVOU), and reduced stochastic model (RSM) for $n=32$ (a), $n=16$ (b), and $n=8$ (c). Note the different scaling for the ordinate in the figures.

The probability density functions of the models are depicted in figure 4.5. Overall, it can be stated that the multivariate OU model performs best, indicating that the probability density distribution of the full model is close to Gaussian. The linearclosure stochastic model and the reduced stochastic model show similar precision. For an averaging interval $n=32$ the reduced stochastic model does better than the linear-closure stochastic model, but its performance worsens for smaller values of $n$ due to the decreased scale separation between the $x$ - and $y$-modes.

4.3. Budget analysis. One advantage of the subgrid-scale parameterization derived by stochastic mode reduction is that the different subgrid-scale corrections can be attributed to different physical processes [16, 17, 21, 11]. By performing a budget analysis for the different subgrid-scale correction terms, we can systematically assess the importance of different physical interactions $[11,10]$. In order to evaluate the contributions of various subgrid-scale corrections, we regroup the different terms in $(3.5)$ as

$$
d x_{i}(t)=\lambda_{b} \sum_{j, k=i-1}^{i+1} B_{i j k}^{x x x} x_{j} x_{k} d t+\lambda_{a}\left(\sum_{j=i-1}^{i+1} M_{i j}^{a} x_{j} d t+\sum_{j=i-\frac{1}{2}}^{i+\frac{1}{2}} P_{i j} d W_{j}^{(1)}\right)
$$


a

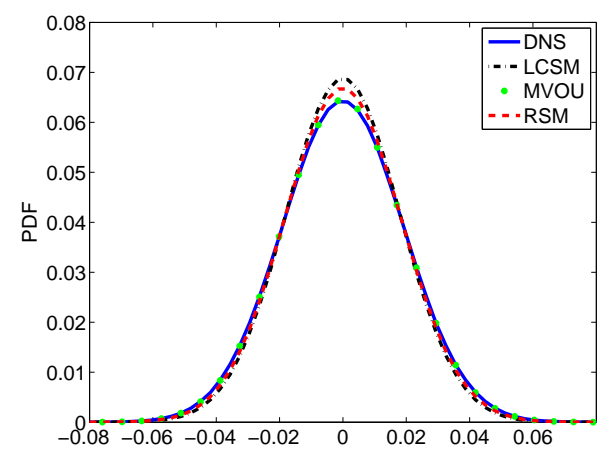

$\mathrm{b}$

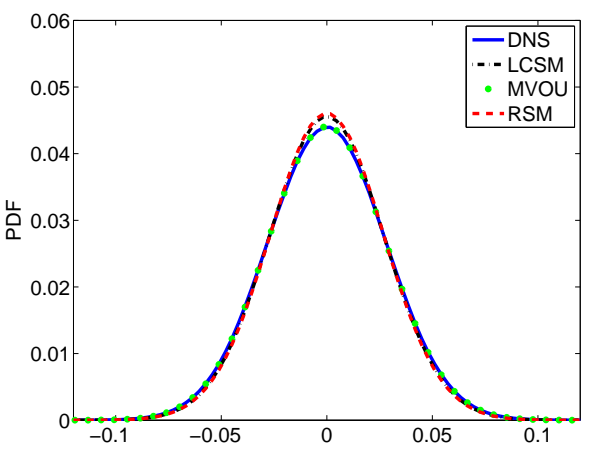

$\mathrm{C}$

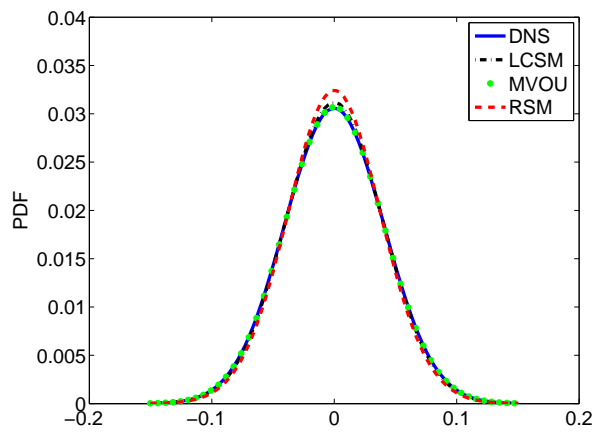

FIG. 4.5. Probability density function of the resolved modes from the DNS, linear-closure stochastic model (LCSM), multivariate OU model (MVOU), and reduced stochastic model (RSM) for $n=32$ (a), $n=16$ (b), and $n=8$ (c). Note the different scaling for the axes in the figures.

$$
+\lambda_{m}\left(\sum_{j=i-1}^{i+1} M_{i j}^{m} x_{j} d t+\sum_{j, k, l=i-2}^{i+2} C_{i j k l} x_{j} x_{k} x_{l} d t+\sum_{j=i-\frac{1}{2}}^{i+\frac{1}{2}} D_{i j}(x) d W_{j}^{(2)}\right),
$$

with $\lambda_{b}=\lambda_{a}=\lambda_{m}=1$ for the complete reduced stochastic model. Interactions involving the terms $B^{x y y}$ and $B^{y x y}$ in the full model are called additive triad interactions; they give rise to linear corrections and additive noise in the subgrid-scale parameterization. We will refer to these subgrid-scale terms as additive corrections, and we place $\lambda_{a}$ in front of them to denote their origin. Multiplicative subgrid-scale correction terms include linear and cubic terms, as well as multiplicative noise. These terms are multiplied by $\lambda_{m}$ and they are due to multiplicative triad interactions involving $B^{x x y}$ and $B^{y x x}$. Finally, we mark the bare truncation term with an $\lambda_{b}$-factor in front of it. The explicit form of the subgrid-scale corrections discussed above can be found in Appendix B.

We perform simulations with a single type of interaction switched on, i.e., just one among $\lambda_{b}, \lambda_{a}$ or $\lambda_{m}$ is $=1$, and all others 0 . The results are summarized in figure 4.6. First, we see that the bare truncation model (BRT), a model without any subgrid-scale corrections, fails completely to reproduce the autocorrelation function and kurtosis of the DNS. Further, a reduced model with only additive triad corrections captures the autocorrelation function and probability density function of the reduced stochastic model, indicating that the additive triads are the dominant interactions. However, by 
a

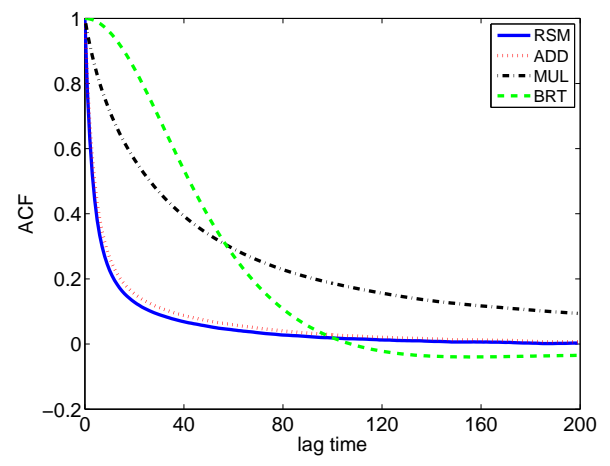

$\mathrm{b}$

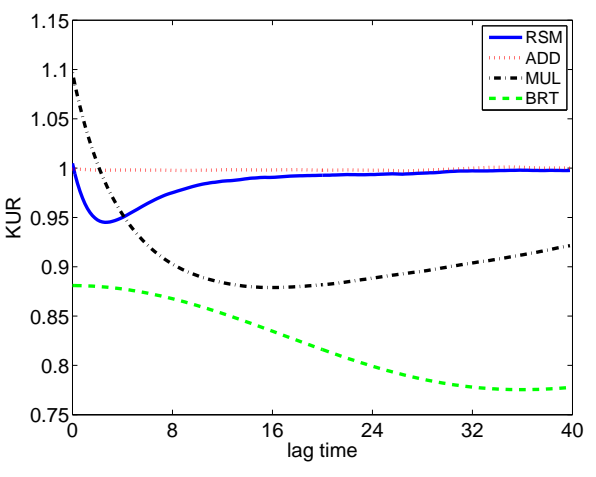

$\mathrm{C}$

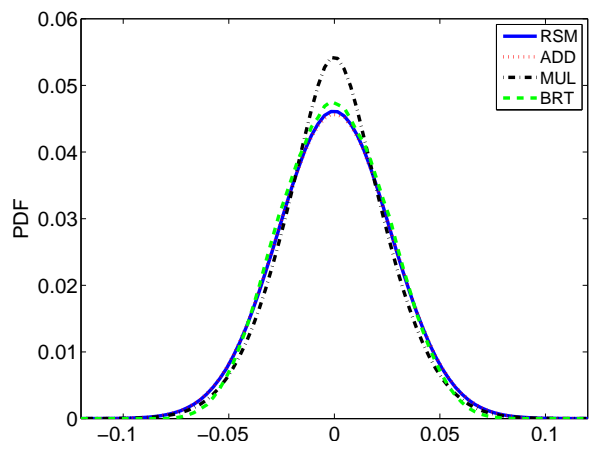

FIG. 4.6. Autocorrelation function (a), kurtosis (b), and probability density function (c) computed with different correction terms in the reduced stochastic model: bare truncation (BRT), additive triads (ADD), multiplicative triads (MUL), and full reduced stochastic model (RSM). All models with $n=16$. See text for an explanation of the different correction terms.

definition such a model does not show any deviations from Gaussianity (since this is a purely Gaussian process); see figure 4.6 (b). The statistics from simulations with two types of interactions are displayed in figure 4.7. We observe that a model including additive corrections and multiplicative ones, i.e. $\lambda_{a}=\lambda_{m}=1$ and $\lambda_{b}=0$, reproduces not only the autocorrelation function and the probability density function of the full reduced stochastic model with $\lambda_{a}=\lambda_{m}=\lambda_{b}=1$, but also the kurtosis. Clearly the closure effectively controls the statistics of the reduced stochastic model.

\section{Summary and conclusions}

We present a framework for the construction of stochastic subgrid-scale parameterizations consistent with the finite-difference discretization of the model equations. The subgrid-scale model is based on the assumption of a time scale separation between resolved and subgrid-scale modes. It assumes further that the fast self-interactions between subgrid-scale modes in the equations for the subgrid-scale modes can be modeled as an one dimensional Ornstein-Uhlenbeck (OU) process. The particular form of the subgrid-scale correction terms is derived in a systematic way from the discretized model by applying the Majda, Timofeyev, and Vanden-Eijnden stochastic mode reduction strategy $[16,17,21]$. The subgrid-scale parameterization is rigorously valid in the limit of infinite scale separation between resolved and unresolved 
a

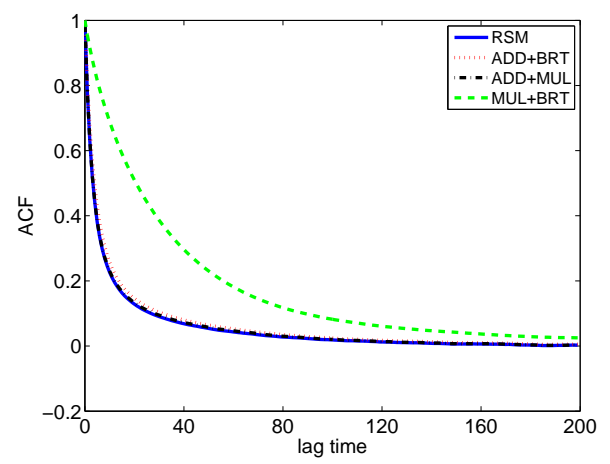

$\mathrm{b}$

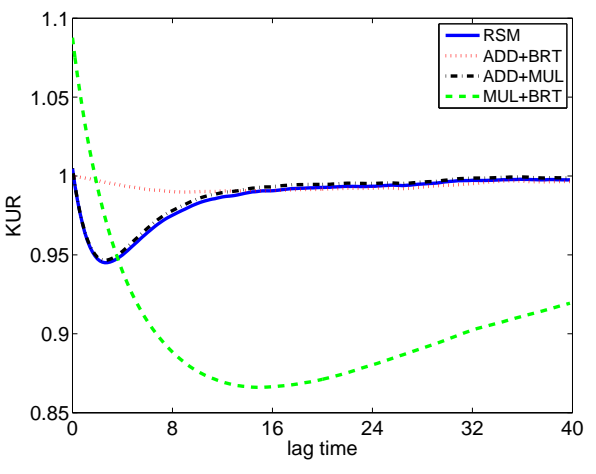

C

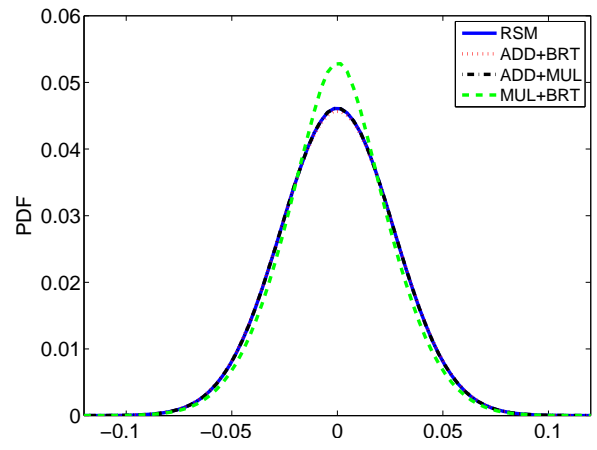

FIG. 4.7. Notation as in figure 4.6.

modes. It includes linear and cubic deterministic corrections, as well as additive and multiplicative noise terms. The subgrid-scale corrections are determined without any regression fitting of the resolved modes, and require only the variance and integrated autocorrelation function of the subgrid-scale modes as input.

As a test model for the new framework we use the inviscid Burgers-Hopf equation. We consider an energy and momentum conserving finite-difference discretization of the model equation [20] and construct for it a stochastic subgrid-scale parameterization. The resulting reduced stochastic model has been compared with the full model (DNS) and with two purely empirical coarse-grained models. The first such model is the multivariate OU model, constructed in such a way as to match the covariance and integrated lagged cross-correlations of the full model. The second empirical model is the linear-closure stochastic model, which incorporates one dimensional OU closure in addition to the non-linear self-interactions of the resolved variables. In order to assess the quality of the different subgrid-scale parameterizations, we performed simulations by varying the number of subgrid-scale modes, which have to be modeled, and comparing the resulting statistics with those from the full model.

All coarse-grained models capture the probability density function from the DNS, with the multivariate OU model performing best. However, the latter model completely underestimates the autocorrelation decay time scale. The linear-closure stochastic model and the reduced stochastic model, on the other hand, can capture the overall decay behavior in the autocorrelation function. Beyond this, the reduced 
stochastic model exhibits several advantages. (1) It captures the autocorrelation function at very small lags. (2) If the oscillations in the autocorrelation function of the DNS are small, the reduced stochastic model captures also the low-frequency envelope. The linear-closure stochastic model, on the other hand, decays always too fast at large lags. (3) The inspection of the lagged kurtosis of the models showed that the reduced stochastic model is able to capture better than the linear-closure stochastic model some weakly non-Gaussian features in the DNS. (4) The statistics of the resolved modes show large changes, in contrast to those of the subgrid-scale modes, when the total number of resolved modes $N_{x}$ is varied in the full model. Thus, the subgrid-scale parameterization of the purely empirical coarse-grained models must be recomputed for each case considered here. However, such recomputation is not required for the reduced stochastic model, since for this parameterization the dependency on $N_{x}$ (and on the averaging interval $n$ ) is known. Of course, for the Burgers-Hopf equation, considered here, one can find a simple scaling relation between the parameters in the purely empirical closures and $N_{x}$, in order to avoid recomputing these parameters. However, this might not be the case if more complex models are considered. (5) Another practical advantage of the reduced stochastic model is that the empirical coefficients entering the closure can be inferred from a shorter time-series since they only depend on the fast subgrid-scale modes. This is in contrast with empirical reduced models (such as the MVOU and LCSM models in (4.1) and (4.5), respectively), where the coefficients are computed from statistics of the slow modes characterized by long decorrelation time scales.

An interesting result was that the statistics of the reduced stochastic model are effectively controlled by the closure. By performing a budget analysis it was found that the linear deterministic term and additive noise are the dominant subgrid-scale corrections in the model. These terms result from nonlinear interactions between subgrid-scale modes. Next in importance come the multiplicative correction terms, followed only by the deterministic terms from the barely truncated model.

One possibility to improve the present approach is by incorporating interactions between different subgrid-scale modes in the OU process from the closure assumption. Work on the Burgers equation with forcing and dissipation suggests that the subgridscale self-interactions can be modeled by an OU process coupling small number of neighboring grid cells [9].

Acknowledgment. We would like to thank Andrew Majda and Christian Franzke for useful discussions. We thank the two reviewers for their comments and suggestions which helped to improve the manuscript. U. A. thanks Deutsche Forschungsgemeinschaft for partial support through the MetStröm Priority Research Program (SPP 1276) and through Grant Ac71/4-1; I. T. thanks the NSF for partial support through grants DMS-0713793 and DMS-1109582.

Appendix A. Interaction coefficients in the discretized Burgers-Hopf equation. The quadratic interaction terms from (2.9), (2.10) are given by

$$
\begin{aligned}
\sum_{j \in s_{x}} \sum_{k \in s_{x}} B_{i j k}^{x x x} x_{j} x_{k} & =-\frac{1}{6 n \Delta x}\left(x_{i+1}^{2}+x_{i} x_{i+1}-x_{i} x_{i-1}-x_{i-1}^{2}\right), \\
\sum_{j \in s} \sum_{k \in s} B_{i j k}^{x y y} y_{j} y_{k} & =-\frac{1}{6 n \Delta x}\left(y_{i r}^{2}+y_{i r} y_{i r+1}+y_{i r+1}^{2}-y_{i l}^{2}-y_{i l} y_{i l-1}-y_{i l-1}^{2}\right), \\
\sum_{j \in s_{x}} \sum_{k \in s} B_{i j k}^{x x y} x_{j} y_{k} & =-\frac{1}{6 n \Delta x}\left(2 x_{i} y_{i r}+x_{i} y_{i r+1}+x_{i+1} y_{i r}+2 x_{i+1} y_{i r+1}\right.
\end{aligned}
$$




$$
\begin{gathered}
\left.-2 x_{i} y_{i l}-x_{i} y_{i l-1}-x_{i-1} y_{i l}-2 x_{i-1} y_{i l-1}\right) \\
\sum_{j \in s_{x}} \sum_{k \in s} B_{i j k}^{y x y} x_{j} y_{k}=-\frac{1}{6 \Delta x}\left(2 x_{\left[\frac{i+1}{n}\right]} y_{i+1}+x_{\left[\frac{i+1}{n}\right]} y_{i}+x_{\left[\frac{i}{n}\right]} y_{i+1}\right. \\
\left.-2 x_{\left[\frac{i-1}{n}\right]} y_{i-1}-x_{\left[\frac{i-1}{n}\right]} y_{i}-x_{\left[\frac{i}{n}\right]} y_{i-1}\right) \\
+\frac{1}{6 n \Delta x}\left(2 x_{\left[\frac{i}{n}\right]} y_{i r}+x_{\left[\frac{i}{n}\right]} y_{i r+1}+x_{\left[\frac{i}{n}\right]+1} y_{i r}+2 x_{\left[\frac{i}{n}\right]+1} y_{i r+1}\right. \\
\left.\quad-2 x_{\left[\frac{i}{n}\right]-1} y_{i l-1}-x_{\left[\frac{i}{n}\right]} y_{i l-1}-x_{\left[\frac{i}{n}\right]-1} y_{i l}-2 x_{\left[\frac{i}{n}\right]} y_{i l}\right), \\
\sum_{j \in s_{x}} \sum_{k \in s_{x}} B_{i j k}^{y x x} x_{j} x_{k}=-\frac{1}{6 \Delta x}\left(x_{\left[\frac{i+1}{n}\right]}^{2}+x_{\left[\frac{i}{n}\right]} x_{\left[\frac{i+1}{n}\right]}-x_{\left[\frac{i-1}{n}\right]}^{2}-x_{\left[\frac{i}{n}\right]} x_{\left[\frac{i-1}{n}\right]}\right) \\
+\frac{1}{6 n \Delta x}\left(x_{\left[\frac{i}{n}\right]+1}^{2}+x_{\left[\frac{i}{n}\right]} x_{\left[\frac{i}{n}\right]+1}-x_{\left[\frac{i}{n}\right]-1}^{2}-x_{\left[\frac{i}{n}\right]} x_{\left[\frac{i}{n}\right]-1}\right) .(\mathrm{A}
\end{gathered}
$$

In the equations above the index $[i / n]$ denotes the coarse cell corresponding to the fine cell $i$ and the index $i r(i l)$ marks the fine cell at the right (left) boundary of a coarse cell

$$
\begin{aligned}
& i r= \begin{cases}\left(\left[\frac{i}{n}\right]+1\right) n-1, & \text { if } i \in s, \\
(i+1) n-1, & \text { if } i \in s_{x},\end{cases} \\
& i l= \begin{cases}\left(\left[\frac{i}{n}\right]\right) n-1, & \text { if } i \in s, \\
i n-1, & \text { if } i \in s_{x} .\end{cases}
\end{aligned}
$$

Appendix B. Subgrid-scale coefficients in the reduced stochastic model. As discussed in Section 4.3, the different subgrid-scale correction terms can be regrouped depending on their origin. The resulting deterministic terms are given by

$$
\begin{aligned}
\sum_{j=i-2}^{i+2} M_{i j}^{a} x_{j} & =\frac{\sigma^{2}}{2 \gamma^{2}} \sum_{j} \sum_{m, n} B_{i m n}^{x y y} B_{m j n}^{y x y} x_{j}, \\
\sum_{j=i-2}^{i+2} M_{i j}^{m} x_{j} & =\frac{\sigma^{2}}{2 \gamma^{2}} \sum_{j} \sum_{k, l} B_{i l k}^{x x y} B_{l j k}^{x x y} x_{j}, \\
\sum_{j, k, l=i-2}^{i+2} C_{i j k l} x_{j} x_{k} x_{l} & =\frac{1}{\gamma} \sum_{j, k, l} \sum_{p} B_{i l p}^{x x y} B_{p j k}^{y x x} x_{j} x_{k} x_{l} .
\end{aligned}
$$

For the explicit computation of the subgrid-scale correction terms we utilized the MATLAB symbolic toolbox, and obtained the following expressions:

$$
\begin{aligned}
\sum_{j=i-1}^{i+1} M_{i j}^{a} x_{j} & =\frac{\sigma^{2} a^{2}}{4 \gamma^{2} n}\left(x_{i-1}-2 x_{i}+x_{i+1}\right)\left(5+\frac{1}{n}\right) \\
\sum_{j=i-1}^{i+1} M_{i j}^{m} x_{j} & =-\frac{\sigma^{2} a^{2}}{2 \gamma^{2} n^{2}}\left(x_{i-1}-2 x_{i}+x_{i+1}\right) \\
\sum_{j, k, l=i-2}^{i+2} C_{i j k l} x_{j} x_{k} x_{l} & =\frac{1}{\gamma} \frac{a^{2}}{n}\left(5 x_{i-1}^{3}+3 x_{i-1}^{2} x_{i}+3 x_{i+1}^{2} x_{i}+5 x_{i+1}^{3}-3 x_{i-1} x_{i}^{2}-10 x_{i}^{3}-3 x_{i}^{2} x_{i+1}\right) \\
& -\frac{1}{\gamma} \frac{a^{2}}{n^{2}}\left(2 x_{i-2}^{2} x_{i-1}+x_{i-2}^{2} x_{i}+2 x_{i-2} x_{i-1}^{2}+x_{i-2} x_{i-1} x_{i}+x_{i-1}^{3}+x_{i+1}^{3}\right.
\end{aligned}
$$




$$
\begin{aligned}
& +x_{i+2} x_{i+1} x_{i}+2 x_{i+1}^{2} x_{i+2}+x_{i+2}^{2} x_{i}+2 x_{i+2}^{2} x_{i+1}-x_{i-1}^{2} x_{i+1}-x_{i-1}^{2} x_{i} \\
& \left.-3 x_{i-1} x_{i}^{2}-2 x_{i-1} x_{i} x_{i+1}-2 x_{i}^{3}-3 x_{i}^{2} x_{i+1}-x_{i} x_{i+1}^{2}-x_{i+1}^{2} x_{i-1}\right)
\end{aligned}
$$

with $a=-1 /(6 \Delta x)$. The subgrid-scale stochastic correction terms are defined by

$$
\begin{gathered}
\sum_{j=i-\frac{1}{2}}^{i+\frac{1}{2}} P_{i j} d W_{j}^{(1)}=\frac{\sigma^{2}}{\sqrt{2 \gamma} \gamma} \sum_{j, k} B_{i j k}^{x y y} d \tilde{W}_{j k}^{(1)}, \\
\sum_{j=i-\frac{1}{2}}^{i+\frac{1}{2}} D_{i j}(x) d W_{j}^{(2)}=\frac{\sigma}{\gamma} \sum_{j, k} B_{i j k}^{x x y} x_{j} d \tilde{W}_{k}^{(2)} .
\end{gathered}
$$

The right hand sides of the last two equations represent the general form of the noise terms after the mode reduction procedure; see [16]. These terms involve independent Wiener processes $\tilde{W}^{(1)}$ and $\tilde{W}^{(2)}$ with the dimension of $N \times N$ and $N$, respectively ( $N$ is the number of subgrid-scale modes). This high dimensionality makes (B.7), (B.8) inappropriate for an implementation. However, all terms on the right hand side of (B.7), (B.8) are associated with a flux either at the left or at the right boundary of the coarse cell $i$. Terms corresponding to the same flux are combined and represented using an effective Wiener process $\left(W^{(1)}, W^{(2)}\right)$ with the low-dimension $N_{x}$. Thus, the explicit form of the noise terms in the subgrid-scale model reads

$$
\begin{aligned}
& \sum_{j=i-\frac{1}{2}}^{i+\frac{1}{2}} P_{i j} d W_{j}^{(1)}= \frac{\sqrt{5} \sigma^{2} a}{2 \sqrt{\gamma} \gamma n}\left(d W_{i+\frac{1}{2}}^{(1)}-d W_{i-\frac{1}{2}}^{(1)}\right), \\
& \sum_{j=i-\frac{1}{2}}^{i+\frac{1}{2}} D_{i j}(x) d W_{j}^{(2)}=\frac{a \sigma}{n \gamma}\left(\sqrt{5 x_{i}^{2}+8 x_{i} x_{i+1}+5 x_{i+1}^{2}} d W_{i+\frac{1}{2}}^{(2)}\right. \\
&\left.-\sqrt{5 x_{i}^{2}+8 x_{i} x_{i-1}+5 x_{i-1}^{2}} d W_{i-\frac{1}{2}}^{(2)}\right) .
\end{aligned}
$$

\section{REFERENCES}

[1] U. Achatz and G. Branstator, A two-layer model with empirical linear corrections and reduced order for studies of internal climate variability, J. Atmos. Sci., 56, 3140-3160, 1999.

[2] U. Achatz and J.D. Opsteegh, Primitive-equation-based low-order models with seasonal cycle. Part I: Model construction, J. Atmos. Sci., 60, 465-477, 2003.

[3] U. Achatz, G. Schmitz, and K.-M. Greisiger, Principal interaction patterns in baroclinic wave life cycles, J. Atmos. Sci., 52, 3201-3213, 1995.

[4] G. Branstator and S.E. Haupt, An empirical model of barotropic atmospheric dynamics and its response to tropical forcing, J. Climate, 11, 2645-2667, 1998.

[5] A.J. Chorin, A.P. Kast, and R. Kupferman, Optimal prediction of underresolved dynamics, Proceedings of the National Academy of Science, 95, 4094-4098, 1998.

[6] A.J. Chorin, A.P. Kast, and R. Kupferman, Unresolved computation and optimal prediction, Commun. Pure Appl. Math., 52, 1231-1254, 1999.

[7] D. Crommelin and E. Vanden-Eijnden, Subgrid-scale parameterization with conditional Markov chains, J. Atmos. Sci., 65, 2661-2675, 2008.

[8] J. Culina, S. Kravtsov, and A.H. Monahan, Stochastic parameterization schemes for use in realistic climate models, J. Atmos. Sci., 68, 284-299, 2011.

[9] S.I. Dolaptchiev, U. Achatz, and I. Timofeyev, Stochastic closure for local averages in the finite-difference discretization of the forced Burgers equation, Theor. Comp. Fluid Dyn., 2012. DOI 10.1007/s00162-012-0270-1 
[10] C. Franzke and A.J. Majda, Low-order stochastic mode reduction for a prototype atmospheric GCM, J. Atmos. Sci., 63, 457-479, 2006.

[11] C. Franzke, A.J. Majda, and E. Vanden-Eijnden, Low-order stochastic mode reduction for a realistic barotropic model climate, J. Atmos. Sci., 62, 1722-1745, 2005.

[12] I. Horenko, S.I. Dolaptchiev, A.V. Eliseev, I.I. Mokhov, and R. Klein, Metastable decomposition of high-dimensional meteorological data with gaps, J. Atmos. Sci., 65, 3479-3496, 2008.

[13] S. Kravtsov, D. Kondrashov, and M. Ghil, Multilevel regression modeling of nonlinear processes: Derivation and applications to climatic variability, J. Climate, 18(21), 4404-4424, 2005.

[14] F. Kwasniok, Reduced atmospheric models using dynamically motivated basis functions, J. Atmos. Sci., 64, 3452-3474, 2007.

[15] A. Majda and I. Timofeyev, Low-dimensional chaotic dynamics versus intrinsic stochastic noise: A paradigm model, Phys. D Nonlin. Phenomena, 199, 339-368, 2004.

[16] A. Majda, I. Timofeyev, and E. Vanden-Eijnden, A mathematical framework for stochastic climate models, Commun. Pure Appl. Math., 54, 891-974, 2001.

[17] A. Majda, I. Timofeyev, and E. Vanden-Eijnden, A priori tests of a stochastic mode reduction strategy, Phys. D, 170, 206-252, 2002.

[18] A. Majda, I. Timofeyev, and E. Vanden-Eijnden, Stochastic models for selected slow variables in large deterministic systems, Nonlin., 19, 769-794, 2006.

[19] A.J. Majda and B. Khouider, Stochastic and mesoscopic models for tropical convection, Proceedings of the National Academy of Science, 99, 1123-1128, 2002.

[20] A.J. Majda and I. Timofeyev, Statistical mechanics for truncations of the Burgers-Hopf equation: A model of intrinsic stochastic behavior with scaling, Milan J. Math., 70, 39-96, 2002.

[21] A.J. Majda, I. Timofeyev, and E. Vanden-Eijnden, Systematic strategies for stochastic mode reduction in climate, J. Atmos. Sci., 60, 1705-1722, 2003.

[22] K. Nimsaila and I. Timofeyev, Markov chain stochastic parametrizations of essential variables, SIAM Mult. Mod. Simul., 8(5), 2079-2096, 2010.

[23] U. Schumann, Subgrid scale model for finite difference simulations of turbulent flows in plane channels and annuli, J. Comput. Phys., 18, 376-404, 1975.

[24] F.M. Selten, An efficient description of the dynamics of barotropic flow, J. Atmos. Sci., 52, 915-936, 1995.

[25] D.S. Wilks, Effects of stochastic parametrizations in the Lorenz'96 system, Quart. J. Royal Meteor. Soc., 131, 389-407, 2005.

[26] C.R. Winkler, M. Newman, and P.D. Sardeshmukh, A linear model of wintertime low-frequency variability. Part I: Formulation and forecast skill, J. Climate, 14, 4474-4494, 2001.

[27] N.J. Zabusky and M.D. Kruskal, Interaction of "solitons" in a collisionless plasma and the recurrence of initial states, Phys. Rev. Lett., 15, 240-243, 1965. 